\title{
Quantification method for timolol in in vivo samples for the development of a new glaucoma drug depot
}

Thomas Eickner*, Institute for Biomedical Engineering, University Medical Center Rostock, Germany, email: thomas.eickner@uni-rostock.de

Franziska Kopp, Rudolf Guthoff, Department of Ophthalmology, University Medical Center Rostock, Germany, email: franziska.kopp@med.uni-rostock.de, rudolf.guthoff@med.uni-rostock.de

Andreas Brietzke, Sabine Kischkel, Stefan Oschatz, Niels Grabow Institute for Biomedical Engineering, University Medical Center Rostock, Germany, email: andreas.brietzke@uni-rostock.de, sabine.kischkel@uni-rostock.de, stefan.oschatz@uni-rostock.de, niels.grabow@uni-rostock.de

Klaus-Peter Schmitz, University Medical Center Rostock, Institute for Biomedical Engineering, Rostock, Germany and Institute for Implant Technology and Biomaterials e.V., Rostock, Germany; email: Klaus-peter.schmitz@uni-rostock.de

Glaucoma is the second most common cause of blindness. The only treatable risk factor is an increased intraocular pressure. Because patients often exhibit a poor therapy adherence, a drug depot consisting of ELA-NCO and hyaluronic acid with timolol maleate as incorporated drug was developed to ensure sustained drug release. This drug depot is formed by in situ polymerisation after application into the subconjunctival space.

To test the in vivo drug release of timolol in serum and aqueous humour, a liquid chromatography mass spectrometry method (LCMS) was developed and tested by spike- and recovery experiments. Therfore, samples of serum and aqueous humour were taken from New Zeeland White rabbits. They were spiked with timolol maleate to a final concentration of $50 \mathrm{ng} / \mathrm{mL}$. Afterwards, the samples were extracted and analysed by LCMS.

The LCMS method developed in this study is suitable to determine the concentration of timolol in a range from 0.5 $5000 \mathrm{ng} / \mathrm{mL}$. The external calibration showed high linearity.

Recovery experiments of timolol from spiked serum and aqueous humour samples, respectively showed no loss of timolol. Hence, the extraction method is robust and able to recover the whole amount of timolol from aqueous humour and serum. 


\section{Novel Test Procedure for Testing Antimicrobially Active Materials}

Alexander Stich, Ostbayerische Technische Hochschule Amberg-Weiden, Germany, a.stich@oth-aw.de Sebastian Buhl, Ostbayerische Technische Hochschule Amberg-Weiden, Germany

Clemens Bulitta, Ostbayerische Technische Hochschule Amberg-Weiden, Germany

Several publications already described the risk of infection via microbially contaminated surfaces for personnel and patients in clinical settings. In addition to the cleaning and disinfection measures, antimicrobial surfaces are becoming an additional factor for improving the safety for patient and personnel.

These antimicrobial coatings are tested for their activity and effectiveness based on an artificial procedure, the standard JIS Z 2801 (or ISO 22196). The test samples are contaminated with a predetermined concentration of bacteria and covered with a sterile film. After incubation at $35^{\circ} \mathrm{C}$ and $>90 \%$ humidity, the remaining and viable germs are measured and compared to non active controls. Due to the artificial structure of this process, however, there is always the question of the validity in the practical application of these surfaces and how strong this germ-reducing effect compared to currently common disinfection measures.

Therefore we developed a more realistic test procedure, were the test samples are inoculated and incubated at standard room conditions. To compare the antimicrobial activity to standard cleaning procedures also disinfection and wipe controls were performed.

The results show that the antimicrobial test patterns we use have a strong efficacy. This could be shown reproducibly both with the standard method JIS Z 2801 as well as with our test procedure. This is evident in the results of test patterns, which show a weak antimicrobial activity in the standard procedure that can no longer be detected under more realistic conditions with our method. We also found that a pure application of disinfectant wipes on contaminated surfaces shows no germ-reducing effect. Therefore manual cleaning by wiping is essential for the decontamination of the surfaces. Based on our findings these antimicrobial coatings are complementary to already existing disinfection measures as an additional useful measure for the safety of personnel and patients. 


\section{Inkjet printing for localized coating and functionalization of medical devices}

Robert Mau, Fluid Technology and Microfluidics, University of Rostock, Justus-von-Liebig-Weg 6, 18059 Rostock, Germany, e-mail: robert.mau@uni-rostock.de

Gerrit Paasche, Thomas Lenarz, Otolaryngology, Hannover Medical School, Carl-Neuberg-Str. 1, 30625 Hannover, Germany, e-mail: paasche.gerrit@mh-hannover.de; lenarz.thomas@mh-hannover.de

Hermann Seitz, Fluid Technology and Microfluidics, University of Rostock, Justus-von-Liebig-Weg 6, 18059 Rostock, Germany, e-mail: hermann.seitz@uni-rostock.de

Inkjet printing has become essential for pharmaceutical research as well as biomedical applications. It is a promising tool to meet future challenges in patient-individual designed pharmaceuticals and implants. In this context, the main areas of use are high-throughput screening (HTS), drug-loaded microparticles, drug formulation and oral dose development, 3D-printing/bioprinting as well as coating of implants. This study deals with the latter. In view of promising applications for localized coating and functionalization of implant surfaces this work shows preliminary results on inkjet printing of the polymer poly(2-ethyl-2-oxazoline) (PetOx), a protein repellent polymer (PRP). To deposit single droplets with small volumes $(\sim 500 \mathrm{pl})$ of aqueous PetOx solution $(50 \mathrm{~g} / \mathrm{l})$, printing parameters were determined for the piezo-driven drop-on-demand inkjet printhead NanoTip J, operating in a Nanoplotter 2.1 (both from GeSiM mbH, Germany). Different printing strategies are demonstrated by varying droplet spacing and drying time while printing on hydrophilic glass substrate. Printing and stacking of almost uniform polymer lines (width $\sim 200 \mu \mathrm{m}$ ) is demonstrated. 


\section{Do 3D-printing materials comply with biocompatibility standards for biomedical application?}

Ariane Leone; Department of cardiac surgery, Ludwig Maximilians University, Munich, Germany; ariane.leone@tum.de;

Nikolaus Thierfelder; Department of cardiac surgery, Ludwig Maximilians University, Munich, Germany; nikolaus.thierfelder@med.uni-muenchen.de;

Stefan Leonhardt; Institute of Medical and Polymer Engineering, Technical University Munich, Germany; stefan.leonhardt@tum.de

Maximilian Grab; (1) Department of cardiac surgery, Ludwig Maximilians University, Munich, Germany; (2) Institute of Medical and Polymer Engineering, Technical University Munich; maximilian.grab@med.uni-muenchen.de;

Marco Tscherner; Gerg Lighthouse GmbH, Baiern/Piusheim, Germany; marco.tscherner@gerglighthouse.de;

Christian Hagl; Department of cardiac surgery, Ludwig Maximilians University, Munich, Germany; Christian.hagl@med.uni-muenchen.de;

Fabian König; (1) Department of cardiac surgery, Ludwig Maximilians University, Munich, Germany; (2) Institute of Medical and Polymer Engineering, Technical University Munich; fabian.koenig@med.uni-muenchen.de

Application of 3D-printing in cardiac surgery is increasing. Yet, informations on biocompatibility of 3D-printing materials are scarce. We examined the biocompatibility of seven materials, currently in use as well as in preclinical testing: MED610, AR-M2, silicone-like AR-G1L and AR-G1H (PolyJet), polymer ABS (Fused Deposition Modeling) and acrylate resins ACR1 and ACR2 (Stereolithography).

Materials were printed as discs (diameter $11.3 \mathrm{~mm}$, thickness $3 \mathrm{~mm}$ ) and analyzed by light and digital microscopy for topographic evaluation. Endothelial cell lines $(n=5)$ were isolated from saphenous veins. Material triplicates were placed onto holders into 24-well-plates. Thus avoiding direct cell-material contact, we examined toxic release. Further, materials were pre-incubated in cell-culture-medium for $1 \mathrm{~d}, 4 \mathrm{~d}, 30 \mathrm{~d}, 90 \mathrm{~d}$. Material eluate could enter the medium which was then added to cells. Cell-exposure was performed for $24 \mathrm{~h}, 48 \mathrm{~h}, 72 \mathrm{~h}$ at $37^{\circ} \mathrm{C}$ with $5 \% \mathrm{CO}_{2}$. Cell proliferation was measured by WST1-assay. Cell morphology was assayed by microscopy, viability by Live/Dead-assay. Moreover, cells seeded directly on materials were examined by scanning electron microscopy.

Upper and lower disc surfaces were not identical but clearly differed in MED610, AR-G1L and AR-G1H. ACR1 and ABS did not decrease cell proliferation below 70\% DIN-standard refered to controls. After 48h, MED610 and AR-M2 decreased proliferation, led to cell clusters and altered morphology. Yet, they did not show negative long-terminfluence $(>30 \mathrm{~d})$. Numerous particles $(<200 \mu \mathrm{m})$ detached from MED610 samples. After 24h, AR-G1L reduced proliferation to $5.75 \pm 2.64 \%$, AR-G1H to $21.5 \pm 8.2 \%$. During long-term-incubation, slightly reduced cytotoxic effects were observed. No cell adhesion was possible on AR-G1L and AR-G1H discs. ACR2 showed mixed results.

According to DIN-standard, ACR1 and ABS can be classified biocompatible. AR-M2 and MED610 were cytotoxic only in short-term-incubation. AR-G1L and AR-G1H can be graded strongly cytotoxic. Material characteristics as the MED610 decay could be attributed to specific production processes. For biomedical use, we recommend careful selection regarding the application period. 


\section{An Artificial Oxygen Carrier Fluid for Oxygenator Performance Tests - Proof of Concept}

Niklas Steuer, Department of Cardiovascular Engineering, Institute of Applied Medical Engineering, RWTH Aachen University, Aachen, Germany, steuer@ame.rwth.aachen.de

Lena Schlotterhose, Department of Cardiovascular Engineering, Institute of Applied Medical Engineering, RWTH Aachen University, Aachen, Germany, lenaschlotterhos@web.de

Peter Schlanstein, Department of Cardiovascular Engineering, Institute of Applied Medical Engineering, RWTH Aachen University, Aachen, Germany, schlanstein@ame.rwth-aachen.de

Thomas Schmitz-Rode, Institute of Applied Medical Engineering, RWTH Aachen University, Aachen, Germany, smiro@ame.rwth-aachen.de

Ulrich Steinseifer, Monash Institute of Medical Engineering and Department of Mechanical and Aerospace Engineering, Monash University, Melbourne, Australia \& Department of Cardiovascular Engineering, Institute of Applied Medical Engineering, RWTH Aachen University, Aachen, Germany, steinseifer@ame.rwth-aachen.de

Georg Wagner, Department of Cardiovascular Engineering, Institute of Applied Medical Engineering, RWTH Aachen University, Aachen, Germany, wagner@ame.rwth-aachen.de

Jutta Arens, Department of Cardiovascular Engineering, Institute of Applied Medical Engineering, RWTH Aachen University, Aachen, Germany, arens@ame.rwth-aachen.de

The design of membrane oxygenators is a complex process. One essential part to evaluate the devices are performance tests which are currently performed in vitro using animal blood. In order to reduce the effort of these performance tests, we propose a technical fluid as a blood substitute. For testing of design iterations, this would also facilitate the use of rapid prototyping materials to which blood often reacts poorly. Hence, the time and cost efficiency of the design process will increase. The proposed blood substitute should have the same characteristics as blood regarding fluid dynamics and gas exchange. Here, a proof of concept of the use of perfluorocarbon (PFC) based emulsions in an oxygenator performance test is presented. These fluids consist of highly viscous droplets in a Newtonian liquid and show high oxygen transfer rates, both similar to blood.

Emulsions consisting of PFC, distilled water, and Pluronic F-68 as emulsifier were manufactured by high-pressure homogenisation. The fluids were characterised regarding droplet size, viscosity, and oxygen transfer rate. Droplet size was measured by laser diffraction spectroscopy and viscosity by means of a cone-plate rheometer. The oxygen transfer rate was characterised in a test loop based on ISO 7199, in which the dissolved oxygen was measured by two clark electrodes. Additionally, the volume fraction of PFCs in the fluids was varied: $20 \mathrm{vol} \%$ and $50 \mathrm{vol} \%$.

The fluid shows a shear dependant viscosity, qualitatively similar to blood. The difference is ascribed to the small droplet size (max $139.3 \mathrm{~nm}$ ). The oxygen transfer rate shows a saturation profile similar to blood and is dependent on the PFCfraction. The results show a high potential for future research, since all characteristics are qualitatively comparable to blood. Our future work will include the adjustment of the PFC fraction and the droplet size to increase the similarity to blood. 


\section{Thermomechanical properties of PEGDA and its co-polymers}

*Natalia Rekowska, Institute for Biomedical Engineering, University Medical Center Rostock, Germany, e-mail: natalia.rekowska@uni-rostock.de

Daniela Arbeiter, Thomas Eickner, Niels Grabow, Michael Teske: Institute for Biomedical Engineering, University Medical Center Rostock, Germany, e-mail: daniela.arbeiter@uni-rostock.de, thomas.eickner@uni-rostock.de, niels.grabow@uni-rostock.de, michael.teske@uni-rostock.de

Jan Konasch, Alexander Riess, Robert Mau, Hermann Seitz, Fluid Technology and Microfluidics, University of Rostock, Germany, e-mail: jan.konasch@uni-rostock.de, alexander.riess@uni-rostock.de, robert.mau@uni-rostock.de, hermann.seitz@uni-rostock.de

Current research activities focus on personalized, comfortable and safe products for systemic or local drug application in patients. Poly(ethylene glycol) diacrylate, is in particular interest as a drug delivery material, as it shows appropriate biological properties such as hydrophilicity and low toxicity. Additionally, as an easily photopoliymerizable compound it can be also utilized for the production of scaffolds with the use of different techniques such as stereolithography. Even though it is often used as a biomaterial or as a copolymer in many photopolymer systems for drug delivery, thermomechanical analysis and basic understanding are rare.

Therefore, we investigated the tensile stress and the glass transition temperature of pure PEGDA and of its copolymers with 1,3-butanediol diacrylate or pentaerythritol triacrylate, as a function of the photoinitiator (PI) or acrylate concentration. Additionally, we demonstrated that the washing procedure decreases the tensile stress values. We showed, that by the means of composing PEGDA with these, it is possible to influence thermo-mechanical properties of the sample.. Our outcomes have revealed, that there is no clear influence of the PI concentration on the thermomechanical properties. However there is an influence of the comonomer concentration. Therefore, it should be possible in future experiments to modify drug release profiles. 